\title{
TOTAL SOLAR ECLIPSE OF FEBRUARY 25
}

\author{
By Prof. F. J. M. STRATTON, O.B.E., F.R.S.
}

\begin{abstract}
A TOTAL eclipse of the sun, with a duration of A totality of about three minutes at the most favourably placed stations, will take place on Feb. ruary 25 next. The eclipse commences at dawn in mid-Atlantic, and the belt of totality reaches land in French Equatorial Africa near Libreville. It then passes across Africa to the Sudan, where EI Obeid and Khartoum are near the central line and Port Sudan is on the southern edge of the belt; crossing the Red Sea and Arabia, where Jeddah is just south of the belt, it passes through Kuwait, Iraq, Iran and Central Asia and ends in Siberia. Basra in Iraq is again just south of the belt.

Originally, three expeditions, spread out from the Sudan to Iran, were contemplated by the Joint Permanent Eclipse Committee, but financial reasons, difficulties of transport and political conditions led to considerable alterations of plans, and in the end only two expeditions are being sent, one from Cambridge and one from the Royal Greenwich Observatory. Generous offers of assistance were received from the Foreign Office, the Services, the Ministry of Supply, the Sudan Government, the Suez Canal Company, and various oil companies (Kuwait, Iraq and Anglo-Iranian). Unfortunately, for the reasons indicated, not all of these could be accepted in the end.
\end{abstract}

The Admiralty has taken full responsibility for the Greenwich expedition, which consists of four parties, two in the Sudan, one in Kuwait and one in Iraq. The parties are under the direction of Dr. R. cl'E. Atkinson, chief assistant of the Royal Observatory. The programme includes the measuring of a long-line geodetic arc between the Sudan and Iraq and a study of the moon's limb profile. The method of observation is by accurately timed cinematographic photographs of the revolving solar crescent as seen at four stations just outside the belt of totality. Two pairs of stations will be established, one near each end of the arc ; each pair will place its stations on opposite sides of the belt.

The Cambridge University expedition, under Prof. R. O. Redman, is being financed by the Government Grant Fund of the Royal Society. It is to be stationed close to Khartoum. Prof. Redman will be studying line profiles in the chromospheric spectrum in continuation of work at the eclipses of 1940 and 1936 . He will be using the Cambridge Observatory Rowland concave grating in the second order, with a dispersion of about $2 \cdot 2 \AA . / \mathrm{mm}$. Dr. H. von Kluber will be carrying out photographic photometry of the corona in polarized light with special attention to the inner corona. Determination will be made of electron density nea the sun's limb. and variations in latitude and over sunspot areas will be looked for. Dr. D. E. Blackwell will be scanning the corona with a lead sulphide cell to get information on differences of intensity distribution within the corona between the infra-red and the region near $\lambda 4000 \mathrm{~A}$.; a study of the mechanism of scattering involved in the corona should follow from the observations. Prof. $H$. Zanstra (Amsterdam) is joining the party and using the Hills quartz spectrugraph to examine the discontinuity in the continuous spectrum of the chromosphere at the head of the hydrogen Balmer series. He has shown that this should give a value of the electron temperature of the chromosphere. Dr.
Houtgast and Mr. de Jager from Utrecht will also join Prof. Redman's party.

The third party from Great Britain originally contemplated was a radio party from the Army Operational Research Group and the Telecommunications Research Establishment. This had to be cancelled in the end, but luckily its programme is largely covered by that of a party from the Naval Research Laboratory, Washington, D.C. In addition to high-speed low-resolution spectra of the sun's limb and high-resolution flash spectra near the limits of the Balmer and the Paschen series, this party is measuring the intensity of radio-frequency radiations at $10 \mathrm{~cm}$. and $8 \mathrm{~mm}$. wave-lengths. Another expedition from the United States is being organized by the National Geographic Society, and part of its programme is an attack on the Einstein deflexion of light, to be carried out by Prof. G. A. van Biesbroeck. Both these parties will be stationed near Khartoum. Prof. J. Hargreaves, of the University of Ottawa, is also bringing an eclipse party and may be at Khartoum or at a high-level station near the Red Sea.

Naturally, the Royal Observatory, Helwan, Egypt, is sending an expedition under Dr. M. R. Madwar. Its programme is a photographic photometric study of the corona and a spectrographic study of coronal lines in the blue-violet and ultra-violet. This party will be in elose touch with a French party under Dr. B. Lyot. An Italian party from the Astrophysical Observatory at Arcetri will consist of Prof. G. Abetti and Drs. A. Colacevick, G. Righini and Fracastoro. Their programme includes a flash spectrum in the ultra-violet with a concave-grating spectrograph and one in the visible region with a plane-grating spectrograph; also coronal photographs, with a long-fucus lens for the inner corona and a short-focus lens for the coronal extensions, and a camera working at $f / 0 \cdot 8$ and spectrograph for the infra-red spectrum of the corona.

A large party under Prof. M. Waldmeier, of Zurich, consisting of Dr. Leutenegger, Prof. Schürer, Mr. Scherrer and Prof. E. Guyot and several others, including two mechanics, is taking out fifteen instruments. Their programme includes the following : structure of the inner corona, photometry and polarization of the corona in the photovisual region with an absolute comparison with the brightness of the solar surface; structure of the outer corona in red light; contour of calcium lines in the outer corona; comparison of the absolute intensities of the continuous spectrum of the corona and of a few emission lines; electron density in prominences and limb darkening on the disk. Most of these parties will be clustered around Khartoum, and it must be hoped that the favourable weather conditions confidently anticipated there at this time of year will be present for the eclipse.

One further expedition is projected-from the Kodaikanal Observatory, India, under Dr. A. K. Das. This party expects to go to Iran or Iraq, more probably the latter country. Nothing has yet been reporter of the plans of the Soviet astronomers in Central Asia.

Mention must also be made of elaborate preparations made for the eclipse by the Joint Commission on the Ionosphere of the International Council of Scientific Unions. In close co-operation with 
the International Union of Radio Sciences, observations of radio noise are to be made at a network of stations, mostly outside the belt of totality but on both sides of it. A study of the behaviour of the $E, F_{1}$ and $F_{2}$ layers during the eclipse will be made. The Department of Scientific and Industrial Research is sending Mr. W. R. Piggott to Ibadan, Nigeria, and Mr. C. M. Minnis to Khartoum.

T'he eclipse will be visible as a partial eclipse in the British Isles about $0900 \mathrm{~h}$. on February 25. The magnitude of the partial eclipse will be about $0 \cdot 11$.

\section{ORIGIN OF THE EARTH}

$\mathrm{G}$ EOPHYSICS has hitherto relied mainly on the principles of mechanics of solids and fluids, assisted by geology and atomic physics, especially in relation to the outer parts of the earth. Prof. Harold C. Urey has now prcduced ${ }^{1}$ a new synthesis largely based on physical chemistry. Long as the paper is, it is very condensed and could have been made into about ten papers. The central principles are as follows :

(a) Kuiper's theory of the origin of the solar system is adopted. This requires the planets to have been derived from much more massive protoplanets, which lost the greater part of their mass in the process.

(b) The planets grew by accretion of solid planetesimals, up to some hundreds of kilometres in diameter. The surface features of the moon are regarded as produced by impact of such planetesimals in orbital motion about the earth.

(c) A discussion of the stability of various chemical compounds under different temperatures and pressures reveals some difficulties about the retention of certain elements, notably nitrogen and carbon. This indicates a high temperature at some stage, but not general fusion.

(d) The planets in consequence are supposed to have started as cold bodies, but to have become hot mainly on account of heat developed in accretion.

(e) 'The earth's central core is identified with iron and the inner core with nickel, and it is held that there is still considerable admixture of iron in the rocky shell to within a few hundred kilometres of the surface.

Whether the conclusions are accepted or not, the arguments are novel and important, especially the chemical ones. In what follows I shall mention some outstanding difficulties.

I am not satisfied by any existing theory of the origin of the solar system. Kuiper's appears to fail on account of the Nölke difficulty, that mass lost by the planets would not have enough angular momentum to leave the system. It must either remain spread through the system, where it would probably be visible and would certainly produce secular perturbations of the planets' orbits that are not observed; or it would be absorbed into the sun and make its rotation too rapid. While, again, accretion may be important, it is not clear that the large planetesimals are possible in sufficient numbers. My original argument against the planetesimal hypothesis was that the planetesimals, if they contributed a large fraction of the planets' masses, must have had a much larger total surface than the planets. Consequently, they would collide with and volatilize one another before the planets could grow appreciably.
The argument was answered by A. L. Parson, who showed that the density of the resulting gas would be greater than the saturation density, so that the gas would at once re-condense into dust. But it is difficult to understand why growth occurs by picking up large planetesimals.

Urey, like Hoyle, regards the lunar maria and craters as produced in the early stages, in this respect differing from Baldwin, the chief protagonist of the meteoric theory. I myself still think a volcanic theory tenable. On an accretion theory Urey, again like Hoyle, shows that the earth would become fluid if the material took less than $10^{5}$ years to collect, but would remain solid if it took longer. Urey prefers the latter alternative, Hoyle the former. For myself, I do not see how the strong concentration of radioactive elements near the surface could have occurred except as a result of general fusion and recrystallization; and I know of no satisfactory alternative to the subsequent thermal contraction as an explanation of mountain formation.

W. H. Ramsey's hypothesis that the earth's core is a high-pressure modification of olivine is rejected by Urey on the ground that if it was correct the mean density of the earth, reduced to zero pressure, would be about $3 \cdot 3$, whereas Urey gets about $4 \cdot 4$. But the latter result is got by extrapolating Bullen's pressure-density relation for the core down to zero pressure. It is no argument against Ramsey, because if he is right there is an intervening discontinuity in the relation, and the extrapolation is illegitimate.

Bernal's explanation of the $20^{\circ}$ discontinuity in seismology as due to a transition from rhombic to cubic olivine is also rejected, because Urey's coworkers have not succeeded in preparing the cubic form of $\mathrm{Mg}_{2} \mathrm{GeO}_{4}$ reported by Goldschmidt, from which Bernal inferred the possibility of cubic $\mathrm{Mg}_{2} \mathrm{SiO}_{4}$ at high pressure. This is an awkward fact; but it is hard to see how Goldschmidt could have been mistaken. Urey proposes instead that the dis. continuity is due to admixture of metallic iron. This, however, would not explain the facts. The funda. mental fact is that the times of seismic waves at distances from about $15^{\circ}$ to $25^{\circ}$ indicate a rapid increase of velocity with depth, starting at a depth that might be $150-500 \mathrm{~km}$. on various interpretations. The increase of density inferred by Bullen is a secondary result. The elastic moduli of iron are not very different from those of olivine, and admixture of iron would reduce the velocities of elastic waves on account of the high density. Consequently if Urey's interpretation was right, the velocities would decrease with depth instead of increasing.

The variation of velocity of seismic waves with depth is smooth from a depth of $800 \mathrm{~km}$. or so until a little outside the.core; but just outside the core the variation becomes much less rapid. Bullen pointed out that this could be explained $b$ admixture of denser material. Urey's notion of admixture of iron would agree here. Urey suggests furner that settling of iron into the core is still going on (he considers that on the whole the planets acquired their silicates first and that iron planetesimals were added later). This would decrease the moment of inertia and prcduce a secular acceleration of the rotation. This would tend to explain the fact that the observed secular accelerations of the sun and moon are not in the ratio predicted by the theory of tidal friction.

The densities of the inner planets had appeared to be consistent with either the iron-core theory or with 